\title{
eJRIEPS
}

Ejournal de la recherche sur l'intervention en éducation physique et sport

13 | 2008

Varia

\section{Enseigner la danse à des adolescents avec autisme : l'ingéniosité des gestes enseignants}

\section{Emmanuelle Roncin et Monique Loquet}

\section{(2) OpenEdition}

Journals

Édition électronique

URL : https://journals.openedition.org/ejrieps/6085

DOI : 10.4000/ejrieps.6085

ISSN : 2105-0821

Éditeur

ELLIADD

\section{Référence électronique}

Emmanuelle Roncin et Monique Loquet, «Enseigner la danse à des adolescents avec autisme : l'ingéniosité des gestes enseignants », eJRIEPS [En ligne], 13 | 2008, mis en ligne le 01 janvier 2008, consulté le 17 juin 2021. URL : http://journals.openedition.org/ejrieps/6085 ; DOI : https://doi.org/ 10.4000/ejrieps. 6085

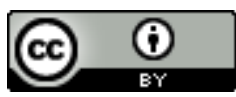

La revue eJRIEPS est mise à disposition selon les termes de la Creative Commons Attribution 4.0 International License. 
Emmanuelle Roncin \& Monique Loquet.

Centre de Recherche sur l'Enseignement, les Apprentissages et la Didactique, IUFM de Bretagne et Université Rennes 2, France

Cette communication a reçu le «Prix du Jeune Chercheur» décerné par l'Association pour la Recherche sur l'Intervention en Sport (ARIS), lors du Colloque International Co-construire des savoirs. La recherche et les métiers de l'intervention dans les activités physiques, sportives et artistiques. Besançon, France, 12 mai 2006.

\section{Résumé}

Cette recherche d'ingénierie didactique (Artigue, M., 1989, 1990) a permis de mettre à jour l'ingéniosité d'un professeur d'éducation physique et sportive à " entrer dans le jeu » des jeunes autistes et à faire face aux résistances dues à leur handicap. Ces résultats nous ont permis de dégager différentes catégories de gestes régulateurs du professeur et d'appréhender l'action de l'enseignant comme un jeu particulier d'interactions médiées par des savoirs qui se forment en situation. Ces gestes reposent d'une part sur un système d'attentes ouvert, très différent des formes scolaires habituelles et d'autre part sur un milieu dans lequel le corps, la proxémie et la voix (et/ou les paroles) de l'enseignant jouent une fonction essentielle.

Notre communication a pour but d'analyser l'activité du professeur d'éducation physique et sportive (EPS) enseignant la danse à de jeunes adolescents atteints d'autisme. Nos recherches sur l'intervention se concentrent sur le thème des liens sociaux que nouent les acteurs en situation. Ce thème est essentiel car sans liens sociaux, il n'y aurait pas de culture humaine ni de transmission culturelle. Ainsi nous nous intéressons à un intervenant en APSA, à ses gestes de régulation et à leur sens émergeant au cœur de l'interaction réelle avec les élèves. Cette attention accordée à l'interaction effective dotée de sens ne signifie pas un retour pur et simple aux sujets (désirs, motifs, sentiments ... relatifs au fait d'enseigner ou d'apprendre) mais un changement d'échelle pour réévaluer la force didactique au niveau des liens ténus, invisibles, indicibles développant la culture 
des hommes (Loquet, M., Roessle, S. \& Roncin, E., 2005). Notre travail s'efforce ainsi d'identifier les « ingrédients " subtils constitutifs de ces liens et de décrire l'ingéniosité in situ du professeur à "entrer dans le jeu » des jeunes autistes et à faire face aux résistances dues à leur handicap. Dans cette perspective nous analysons les relations formées autour des savoir-faire ou capacités lors des différentes séances, sans vouloir décrire ici les savoir-faire ou capacités développés avec et par ce public particulier ni le déroulement des séances typiques d’EPS spécialisée pour les adolescents avec autisme.

\section{Problématique}

De nombreuses pratiques visent à améliorer la vie quotidienne des personnes avec autisme, qu'elles soient en relation ou non, avec le développement de la motricité. Toutefois les pratiques fondées sur une problématique didactique, sont inexistantes. Le terme didactique est employé ici dans un sens très général, en substitution à « processus d'enseignement-apprentissage » pour souligner l'essence de l'activité humaine qui consiste à enseigner quelque chose à quelqu'un d'autre, ou bien, apprendre quelque chose grâce à quelqu'un d'autre. Nous étudions donc un processus qui serait incompréhensible, pour le professeur comme pour les élèves, s'il n'était référé à ce quelque chose à enseigner ou à apprendre. Ce quelque chose en APSA concerne les " savoirs du corps " (Loquet, à paraître). Par action didactique en APSA, il convient alors d'entendre: ce que font les individus dans des institutions où l'on enseigne et où l'on apprend des « savoirs du corps ».

Notre recherche d'ingénierie didactique a pour but de concevoir, réaliser et évaluer un enseignement de la danse pour des adolescents avec autisme. Le syndrome autistique désigne un phénomène complexe composé de traits caractéristiques pathologiques ou déviants du développement normal. On observe des problèmes de communication verbale et/ou non verbale au niveau quantitatif et qualitatif. Pour une meilleure compréhension des objectifs assignés à l'ingénierie, nous souhaitons préciser les difficultés majeures que rencontrent ces jeunes avec autisme: (i) les difficultés en relation avec le corps: les personnes avec autisme établissent rarement le contact physique, « paraissant même l'éviter, le refuser, le fuir. II peut être agacé, anxieux, inquiet lorsqu'il est sollicité ॥. (Barthelemy, C., Hameury, L. \& Lelord, G., 1995, 24). Une autre cause de frustration chez ces jeunes avec autisme serait leur difficulté à sentir, ressentir leurs frontières corporelles, les limites de leurs corps. Pour les sujets autistes et psychotiques, « le corps ne semble pas avoir pris de coordonnées humaines, il reste proche de la chair ». (Brunet, F. \& Caouette, M., 1995, 115); (ii) les difficultés en relation avec la communication, 
l'expression des émotions, des sentiments : Une personne avec autisme « évite le contact visuel, son regard n'est pas utilisé pour attirer l'attention, il est (...) « périphérique, ou bien « vide », « transparent ». (Barthelemy, C., Hameury, L. \& Lelord, G., 1995, 24)

Cette difficulté à entrer en contact visuel, d'attention1, d'attention sélective2, d'attention conjointe ou d'attention partagée3 est un obstacle à la construction d'une théorie de l'esprit et d'un langage corporel adapté lié aux émotions. La théorie de l'esprit est le fait de «prédire les relations existant entre des états de fait externes et des états d'esprit internes ». (Frith, U., 1992, 258). Pour Jordan Powell (1997), «l'un des mécanismes fondamentaux qui permettent aux comportements sociaux de se développer est celui du contact oculaire » (21). Le contact se faisant entre les yeux et le visage du partenaire (pas seulement le regard). "C'est la déviance dans la qualité réciproque du contact visuel qui distingue les autistes des autres personnes, et pas seulement l'évitement du regard », l'enfant autiste « ne sait pas se servir du regard pour communiquer »(22). Une personne ne focalisant pas son attention sur les bons stimuli ne pourra faire les bonnes associations d'idées, d'images, les bonnes relations de causes à effet. Une personne dite normale a un «besoin d'obtenir une structure cohérente à partir d'informations disparatres » contrairement aux personnes autistes (Frith, U., 1992, 258). De façon compréhensive, le manque de contact social limite les occasions de capter des normes concernant la manifestation des émotions. Ainsi les personnes autistes « éprouvent bel et bien des émotions : colère, peur, joie, tristesse »(Buten, H., 2003, 41), mais la manière qu'ils ont de l'exprimer corporellement est souvent inadéquate ou parfois exacerbée comparativement à la plupart d'entre nous. Ils peuvent être agressifs envers eux même (se frapper, se mordre) ou envers les autres (mordre, pincer, griffer, taper...).

L'ingénierie, en gérant l'antagonisme danse/autisme, est conçue pour développer un apprentissage collectif (et pas seulement individuel) de conduites sociales (et pas seulement motrices). Elle vise à réhabiliter l'apprentissage de la danse vue comme pratique socioculturelle, alors que l'institution tend à utiliser des formes d'apprentissage basées quasi exclusivement sur les codes techniques identifiés en danse et appliqués aux situations singulières. L'ingénierie destiné aux jeunes autistes poursuit un double objectif : (i) développer l'aptitude à communiquer et agir de façon concertée ; (ii) incorporer des savoirs sociaux liés à l'expression non verbale des émotions et sentiments propres à la

\footnotetext{
${ }^{1}$ L'attention peut être mesurée par le regard ou même plus sommairement par la position de la tête.

${ }^{2}$ L'attention sélective est le fait de diriger son attention (ex. : pointer du doigt), mettre en évidence un élément clé.

${ }^{3}$ La fonction clé de l'attention partagée ou conjointe est de tendre à repérer l'objet d'intérêt partagé par soi et une autre personne, de construire une représentation triadique. (Baron-Cohen, S., 1995)
} 
danse. Pour cela nous travaillons la conscience du corps à partir des contacts et des porters. Ce travail sur ce double objectif permet d'agir sur les deux difficultés majeures développées ci-dessus. Engager ces jeunes dans l'activité danse, c'est-à-dire dans une activité de communication et de transmission de « message », nous amène à définir l'acte de « danser » comme suit : danser consiste à produire des formes corporelles expressives (pour soi) et signifiantes (pour les autres) au regard d'un système sémiocinétique où le mouvement a valeur de signe.

Notre objet n'est pas la description des situations produites par l'ingénierie mais celle des techniques et gestes que réalise effectivement le professeur d'EPS, collaborateur de l'ingénierie, lorsqu'il intervient en situation. Dans ce contexte in situ, l'étude des techniques et gestes du professeur consiste à rendre compte des manières d'accomplir tel ou tel type de tâches de transmission, auprès des adolescents avec autisme dans une institution para scolaire - c'est-à-dire dans des situations et un environnement particuliers d'APSA qui actualisent et donnent forme à ces tâches. Dans notre étude, les situations dispensées par l'institution concernée (centre spécialisé pour jeunes handicapés) sont vues comme « non institutionnellement » porteuses d'une intention enseignante (au sens de non dispensées par l'Ecole) : ce sont des situations où les interactions professeur/élèves sont en grande partie contingentes (Loquet, M., Roncin, E. \& Roessle, S., 2006). Dans les relations didactiques traditionnelles, comme c'est le cas en classe d'EPS, les échanges autour des objets visés reposent sur un système de communication enseignant/élève(s) qui permet de partager des actions et informations sur les actions. Ces échanges supposent la présence minimale de comportements « altruistes » chez l'élève. Que dire des relations à visée didactique où les enfants semblent effectivement tirer parti des informations fournies par l'enseignant, mais sans en donner (jamais ou rarement) eux-mêmes, comme c'est le cas des jeunes autistes. L'étude de ces situations rapportées aux savoirs corporels qu'elles mettent néanmoins en œuvre et aux formes de leur transmission, présente un grand intérêt: la définition des techniques et gestes didactiques est nécessairement poussée plus avant, car c'est dans l'événement qu'il faut pouvoir caractériser la manière dont les apprentissages et l'enseignement émergent. Faire accéder à la visibilité les techniques et gestes de l'intervenant, c'est les traiter non comme un donné (qu'il s'agirait de prescrire) mais comme une construction (qu'il s'agit de décrire) : aboutissement d'une inter-activité élève(s)-enseignant où l'ingéniosité pratique, dont ce dernier fait preuve, occupe une importance capitale. 


\section{Cadre Théorique}

Notre cadre théorique appartient au champ de la didactique auquel notre recherche emprunte les concepts de milieu et de contrat didactiques (Brousseau, G., 1998), tout en s'inspirant des travaux récents de Perrin-Glorian\& Hersant (2003) sur les niveaux de milieu. "Ces concepts de milieu et de contrat sont essentiels dans la théorie des situations et constituent deux versants complémentaires de la situation didactique puisque celle-ci se compose d'une situation adidactique dans lequel le professeur n'intervient pas au niveau des connaissances et des savoirs et d'un contrat didactique dans lequel elle est plongée, et c'est le milieu qui caractérise la situation adidactique ; si le milieu change, la situation change. En effet, le sujet de la situation adidactique peut être vu comme un système de connaissances qui agit sur un milieu antagoniste ; le milieu réagit aux actions du sujet par des rétroactions que le sujet interprète avec ses connaissances. L'apprentissage se produit à travers l'interprétation des effets des actions sur le milieu, par l'adaptation du sujet au milieu » (Perrin-Glorian, M-J., Hersant, M., 2003).

Le «jeu » didactique4 avec les adolescents avec autisme est régi d'une part, par le contrat didactique et d'autre part, par une mise en place ou plutôt une bascule vers un milieu antagoniste et rétroactif. Nous parlons de bascule dans le sens où le professeur n'est pas le seul à décider du passage vers un milieu dit antagoniste et rétroactif. II doit évidemment mettre en place des éléments du milieu, mais ce passage vers ce milieu ne peut se faire que si les adolescents acceptent de rentrer dans l'action souvent chargée d'émotions. Ce contrat et milieu peuvent être considérés comme étant deux phases successives. On instaure un contrat dans la classe avant de placer les élèves dans un milieu antagoniste et rétroactif. Durant les séances le professeur tente continuellement de faire basculer les jeunes vers ce milieu (bascule de type topogénétique). Compte tenu des difficultés rencontrées par ces jeunes, une grande partie des situations font partie du contrat didactique et se trouvent être des situations didactiques. Le contrat est comme l'a défini Brousseau, « un système d'habitude, engendrant un système d'attente », le contrat permet ainsi la mise en place de règles, de fixer des habitudes grâce à une répétition des situations. «En fait le contrat est une forme de définition d'une situation didactique. Elle lui est équivalente, mais elle permet de dresser un inventaire des contrats suivant la répartition des responsabilités entre l'enseignant et l'élève » (Brousseau, G., 2002). Le fait de répéter à plusieurs reprises les mêmes situations, des gestes qui appartiennent à ces

\footnotetext{
${ }^{4}$ La notion de « jeu » est comprise au sens développé par Sensevy (2006) : « La notion de jeu peut fournir un modèle pertinent pour mettre en évidence certains aspects du monde social et de l'activité humaine. Le modèle du jeu présente en effet le mérite de souligner les aspects affectifs de l'action (l'investissement dans le jeu) et ses aspects effectifs, pragmatiques (quand et comment gagne-t-on ?) ».
} 
dernières, est une chose incontournable et très importante pour espérer fixer un comportement chez ce public (qui peut du jour au lendemain réagir d'une toute autre manière). Malgré le fait que la majeure partie des situations permet de fixer le contrat, ce système d'habitude est à chaque séance actualisé par une ou des situations particulières, spécifiques, chargées d'une nouvelle capacité à développer. Par exemple à chaque séance lors de l'échauffement les contacts vont être de plus en plus intrusifs, on va passer de contacts main - main à des contacts main - visage par exemple. Or pour que cette actualisation du contrat didactique se fasse, une mise en place de milieux et, de ce fait, de situations sont à penser.

A un premier niveau de milieu, l'adolescent est confronté au « milieu matériel » (voir souvent humain, corporel dans notre ingénierie), dans ce cas nous sommes dans une « situation objective » où les connaissances personnelles, privées mais anciennes sont activées. L'utilité de ces situations est justement de répéter les connaissances abordées ou acquises aux séances précédentes, pour les fixer.

A un second niveau, l'adolescent est placé dans un « milieu objectif », dans lequel la « situation de référence » permet de faire émerger chez les élèves des connaissances en jeu, connaissances nouvelles.

Le troisième niveau avec lequel les adolescents peuvent être confronté est le « milieu de référence ». II place les adolescents dans une " situation d'apprentissage », situation adidactique où ils sont réflexifs (et actifs dans notre activité danse) par rapport au milieu. Dans ce cas de figure, ce milieu peut être défini comme étant antagoniste et rétroactif. « Le milieu est le système antagoniste de l'actant. Dans une situation d'action, on appelle "milieu" tout ce qui agit sur l'élève ou / et ce sur quoi l'élève agit. L'actant est " ce " qui dans le modèle agit sur le milieu de façon rationnelle et économique dans le cadre des règles de la situation. En tant que modèle d'un élève ou plus généralement d'un sujet, il agit en fonction de son répertoire de connaissances. La structuration du milieu didactique de l'élève fait apparaître un emboîtement de situations correspondant à des projets distincts et dont chacune sert de milieu à la suivante »(Brousseau, G., 2002).

\section{Méthodologie}

Le corpus principal est composé de huit séances où trois adolescents autistes évoluent avec un professeur impliqué dans la construction raisonnée des situations qu'il devra 
ensuite animer. Chaque séance d'une heure est filmée, transcrite verbalement et de façon proxémique5, et cernée par différents entretiens.

- Entretiens ante séance et post séance

Nous avons procédé à huit entretiens ante séance et huit entretiens post séance qui permettent d'établir une confirmation d'indices et de traces utiles à notre questionnement et de répondre à certaines questions ( ou à émettre des hypothèses).

Les entretiens ante séance concernent le projet de l'enseignant, projet réalisé entre l'enseignant et le chercheur. Les entretiens post séance discutent de ce qui s'est passé pendant les séances, et s'intéressent particulièrement aux difficultés et obstacles rencontrés par les élèves.

- Entretien protocole

Un entretien dit protocole questionne le professeur sur les séances (avec vidéo) et s'appuie sur ce qui a été dit lors des autres entretiens. Pour l'analyse nous opérons des allers-retours entre les séances et les entretiens de la manière suivante (figure 1).

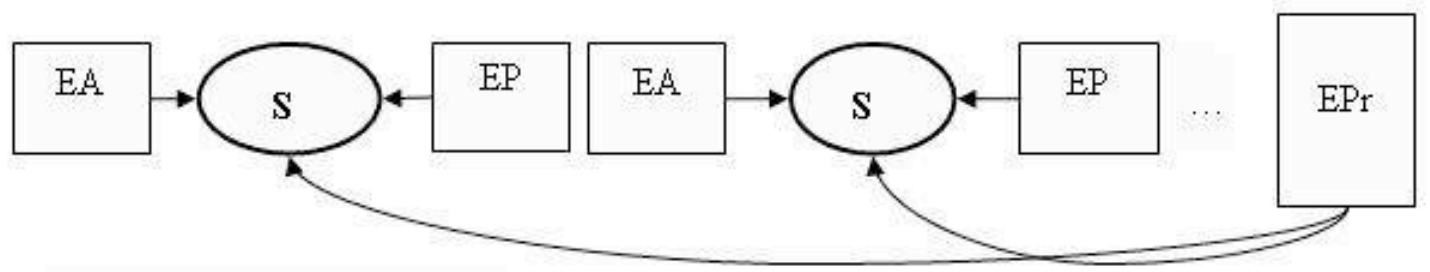

\footnotetext{
Entretien ante séance : $\mathrm{EA}$

Entretien post séance : EP

Entretien protocole : $\mathrm{EPr}$

Séance : $\mathbf{S}$
}

Figure 1 : Schéma du corpus

La méthode d'analyse qui suit s'inspire des algorithmes de Sensevy (cité par Loquet, M. et al., 2006).

\section{- Sélection des séquences}

A partir du visionnage et de la transcription des huit séances, nous avons retenu des épisodes potentiels («E0 »). II concerne des séquences sélectionnées par rapport à leur densité didactique (c'est-à-dire transformatrice ou prometteuse de la transformation des élèves) présentant un condensé d'interactions.

\footnotetext{
5 L'analyse proxémique consiste en une description prenant en compte les gestes, les distances et les regards. Cette description nous a semblée essentielle puisque les autistes avec lesquels nous travaillons ne parlent pas ou très peu. De plus nous avions fait l'hypothèse que cette proxémie devait avoir un impact sur l'avancée des savoir-faire ou capacités.
} 


\section{- Vue synoptique}

Des tableaux synoptiques (courts et larges) des huit séances ont été réalisés pour se familiariser au mieux avec celles-ci. Ces tableaux s'appuient sur les «tours de parole » et « tours d'action » de chacun des acteurs. Ils rendent compte de manière plus détaillée de la temporalité linéaire de l'action découpée selon des critères relativement neutres : modalités de travail (grand/petit groupe, individu), tâche effective, indice de coupure à l'initiative d'un acteur (modes de transition d'une phase à l'autre, d'un jeu à l'autre). Ce découpage (dans les termes des professionnels) scande la succession chronologique des activités occupant collectivement l'enseignant et les élèves.

\section{- La chaîne du récit}

Suite à ces tableaux, des « récits » des séances ont été produits. Ils permettent la mise en valeur de la circulation des savoirs et des chevauchements.

De ces tableaux et récits, des épisodes pertinents « $\mathrm{E} 1$ » (qui pour la plupart nous avaient semblé potentiels) ont émergé. Nous avons ensuite réalisé deux types de narration des ces épisodes « $E 1$ » pour se focaliser de manière encore plus pointue sur ces derniers. De ces zooms sur les « E1 » ressortent:

(i) pour la première narration, des micro-épisodes ${ }^{6}$, des hypothèses sur certains comportements problématiques ou non des adolescents, sur des bascules souvent de type topogénétique. Ces hypothèses ont servi à alimenter le questionnement du professeur lors de l'entretien protocole.

(ii) pour la seconde narration, des gestes qu'utilise le professeur pour faciliter l'entrée ou le maintien en activité des jeunes avec autisme.

\section{- Interprétation des épisodes pertinents}

Nous passons à la double analyse, épistémique et didactique, qui permet de faire passer l'épisode «E1» en «E2», processus qui exige une montée en puissance de la théorisation didactique. L'analyse épistémologique nous conduit à mettre en valeur de façon précise les enjeux de ces épisodes pertinents «E1 » tout en étudiant les possibilités d'action offertes aux élèves pour transformer leur motricité. L'analyse didactique - définie dans notre cas au regard du "comment " le professeur va gérer, tenir le milieu et le contrat didactique (avec ces différents niveaux de milieux) - a permis de mettre à jour l'ingéniosité des gestes et techniques de ce professeur d'EPS à dépasser le handicap et à faire avancer les savoir-faire ou plus précisément les capacités chez ces jeunes autistes.

\footnotetext{
${ }^{6}$ Les micro-épisodes sont des bascules (de type topogénétique ou mésogénétique) qui surviennent lors des épisodes pertinents «E1 ». Elles peuvent provoquer des changements de situations, de phases.
} 
Cette transformation en épisode " E2 » accomplie, des généralisations entre les différents épisodes «E2 » peuvent être faites. Nous avons pu mettre en évidence des comportements, des techniques spécifiques et récurrentes, quasiment toujours accompagnés de gestes, qu'use le professeur pour maintenir le jeu didactique.

\section{Résultats et interprétations}

Les principaux résultats qui font suite à la transformation des épisodes «E1 » en « $E 2$ », mettent en évidence chez le professeur observé une certaine adresse et inventivité : (i) à transformer les milieux pour faire avancer les savoir-faire ou capacités des adolescents ; (ii) à jongler avec une multitude de gestes que l'on peut qualifier de facilitateurs de l'appropriation de nouvelles capacités.

Dans un premier temps nous identifions quels sont ces gestes enseignants, puis nous mettons en évidence une technique qui nous a semblé ingénieuse pour faciliter l'enseignement voir l'apprentissage.

\section{Différents gestes du professeur, la « grammaire gestuelle »}

Les gestes enseignants se distinguent en deux catégories (schématisés en figure 2) : (i) les gestes-contacts, où le professeur entre en contact physique avec les adolescents, par pression, effleurement, etc. ; (ii) les gestes sans contact où seul le mouvement et la parole existent, aucune entrée dans l'espace intime n'est créée par le professeur.

\section{Les gestes contacts}

Parmi les gestes-contacts, nous relevons cinq différents types de gestes avec le cinquième qui se démultiplie en trois sous-types :

les gestes de guidance, ou gestes du professeur qui vont mener physiquement, conduire le ou les adolescents jusqu'à une position précise ;

les gestes de maintien, ou gestes qui permettent de "bloquer », arrêter une posture, un contact, soit suite à un geste de guidance, soit lorsqu'un adolescent a réaliser une posture ou un contact que le professeur veut reprendre, veut fixer (ces gestes de maintien sont indispensables pour contrecarrer les temps de latence);

les gestes de sécurisation ou gestes effectués par le professeur à la place qu'il va prendre pour rassurer un ou plusieurs adolescents. Le professeur va, souvent lors de ces gestes, accepter une grande proximité que lui a demandé un adolescent (comme par exemple lorsque le professeur accepte qu'un adolescent s'assoit sur ses jambes, ses genoux) ; les gestes de préparation, ou gestes qui permettent d'habituer les adolescents aux contacts, aux autres. Le professeur va donc entrer en contact avec un ou des adolescents, 
soit avec une ou deux mains (tel un holding), soit avec une partie du corps pour familiariser les adolescents avec la proximité, le contact physique d'autrui ; les gestes d'ouverture (à l'autre), qui comprennent les gestes plus précis suivants : les gestes des possibles, ou gestes par lequel le professeur touche à plusieurs endroits le corps d'un adolescent avec qui il faut entrer en contact lors d'une tâche dont c'est le but (comme lorsqu'il s'agit d'inciter les jeunes autistes qui doivent toucher cet adolescent d'accepter d'entrer en contact avec au moins un endroit présenté par le professeur). Les adolescents peuvent en effet refuser le contact corporel parce que la partie concernée (si le professeur n'en propose qu'une seule), est trop intrusive, ou trop difficile d'accès (s'il faut s'introduire au sein même du groupe pour accéder à la partie du corps désignée par le professeur);

les gestes d'attention, lorsque le professeur touche, le plus souvent le bras, l'épaule ou le torse d'un adolescent, pour qu'il soit attentif à ce qu'il va montrer, pour qu'il regarde ce qui va se passer ;

les gestes combinés lorsque le professeur utilise indépendamment l'une de l'autre, les deux mains et/ou deux gestes (exemples : une main $\rightarrow$ geste de préparation et une main $\rightarrow$ geste d'accompagnement; une main $\rightarrow$ geste d'attention et une main $\rightarrow$ geste de démonstration.

\section{Les gestes sans contact}

Parmi les gestes sans contacts, nous relevons au moins quatre différents types de gestes avec le quatrième geste qui se subdivise en deux sous-types :

Les gestes d'accompagnement, ce sont des gestes ou paroles que le professeur dit ou fait pour accompagner les actions du ou des adolescents. II n'y a pas de contact direct entre le professeur et les adolescents (les regards peuvent être considérés comme des gestes d'accompagnement) ;

les gestes de démonstration, lorsque le professeur montre à un ou plusieurs adolescents la posture, la mimique, le contact à réaliser. Ces gestes de démonstration peuvent de plus être maintenus pour faciliter l'assimilation de la démonstration (ce qui est important face au problème des temps de latence). Ces gestes peuvent aussi être accompagnés de gestes d'accompagnement (parole, regard); les gestes de mimétisme, lorsque le professeur imite un mouvement ou une posture d'un adolescent pour créer ou préserver l'attention d'un ou plusieurs adolescents et pour fixer l'action, c'est-à-dire instituer (positivement) l'action de l'adolescent ; 
les gestes de mimétisme, ce sont des gestes, des postures d'adolescents que le professeur va imiter, reproduire pour créer ou préserver l'attention d'un ou plusieurs adolescents et pour fixer l'action, c'est à dire statuer (positivement) l'action du jeune.

les gestes d'ouverture : les gestes de mise à distance présentent deux cas :

Le professeur s'éloigne des adolescents soit pour créer une situation de rapprochement (assez rare), soit (surtout) pour ne pas court-circuiter une relation qui s'est créée entre des adolescents ;

Le professeur s'éloigne d'un adolescent « acteur » mais rester dans son champ de vision, ceci au profit d'une relation entre cet adolescent acteur et un autre adolescent.

Adolescent « acteur » : ea

Adolescent : e

Le professeur : $\mathrm{P}$

: déplacement du professeur

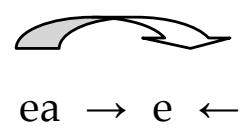

$\mathrm{ea} \rightarrow \mathrm{e} \leftarrow$

Enfin les gestes de félicitation n'apparaissent dans aucune des deux catégories car ces gestes peuvent être exécutés à distance (applaudissements) ou en entrant en contact avec un jeune (taper dans la main). Ce sont des renforcements positifs (physique et/ou oraux) qu'émet le professeur à l'égard des adolescents, comme les applaudissements, serrer la ou les mains...

Ces gestes ont une fonction didactique nécessaire pour enseigner in situ, ils aident le professeur à: (i) mettre en place des règles, des habitudes, faire accepter certaines situations dites didactiques (la cohésion de groupe et/ou l'avancée des savoirs en tant que capacité), de ce fait on peut dire qu'ils permettent de créer, maintenir le contrat didactique qui peut être qualifié de forme(s) d'interactions; (ii) faire basculer les situations didactiques en situations a-didactiques, en se servant de gestes optimisant la relation entre deux ou trois adolescents. 


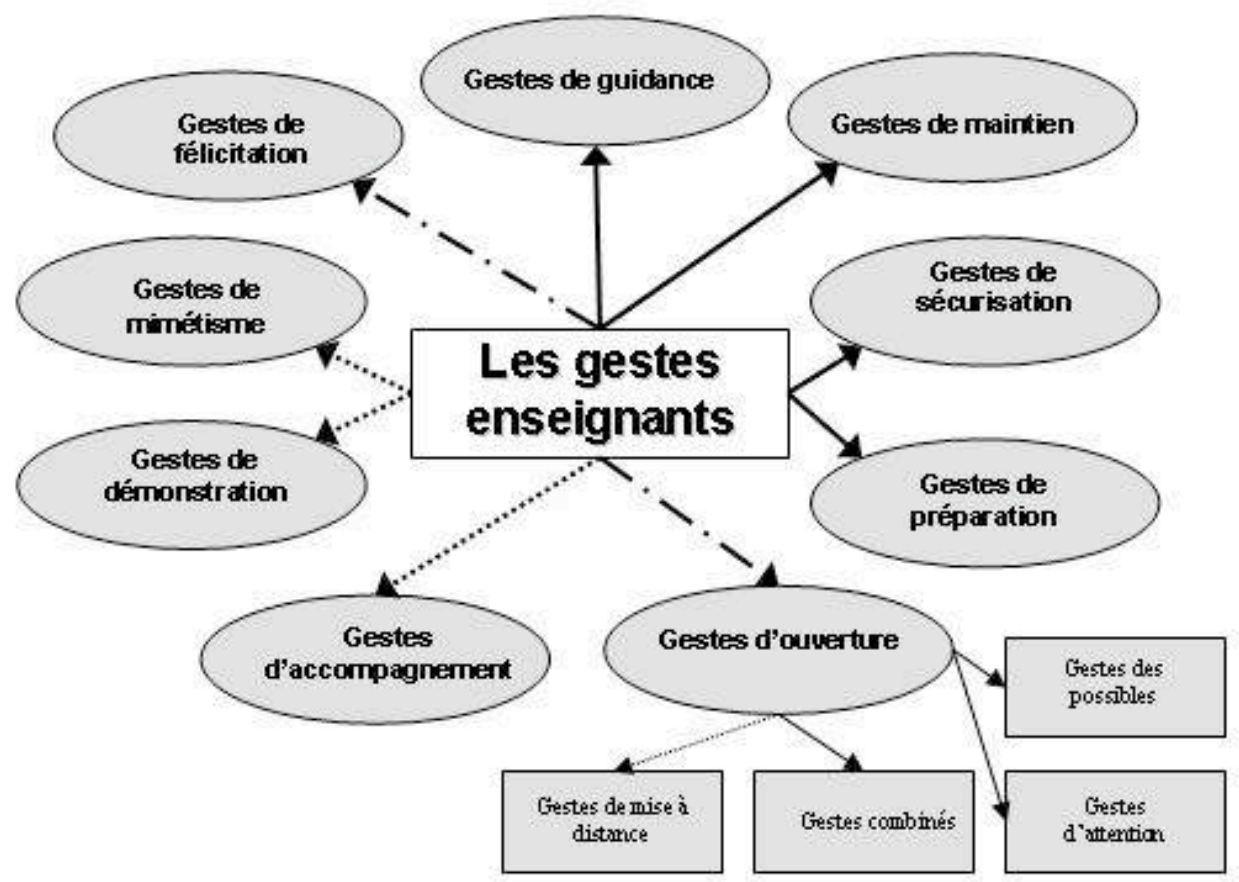

$\longrightarrow$ gestes contacts

$\rightarrow$ gestes sans contact

$\longrightarrow$ gestes avec ou sans contact

Figure 2. Récapitulatif des différentes gestes enseignants

Les techniques ingénieuses du professeur

Le professeur nous a montré une grande habileté à transformer les milieux pour faire avancer les savoir-faire ou capacités des adolescents. Nous allons présenter un exemple de ce qu'on a appelé technique ingénieuse. Cet exemple est accompagné de la figure 3, ainsi les numéros qui se dégagent du texte sont à joindre aux numéros de cette figure. Ce qui apparaît en gris clair sont d'autres ingéniosités observées, mais nous ne les développerons pas dans cette communication.

D'une manière récurrente, les observations mettent en évidence que si le professeur ne dispose pas de la cohésion du groupe au début d'une situation [1] (par exemple enseignement de postures ou contacts nouveaux en relation avec l'émotion tristesse), il va guetter les opportunités didactiques, pour essayer de regrouper les élèves ce qui lui permettra par la suite de revenir sur les contenus liés à la danse de façon collective. Dans ce cas de figure, le professeur pourra utiliser l'isolement d'un adolescent [2], par rapport au groupe, pour créer ou plutôt mettre en valeur un « milieu matériel » en quelque sorte proposé par l'élève solitaire. De là le professeur va user de différents gestes [3] tels que des gestes d'accompagnement, de guidance, de sécurisation, pour faire entrer les autres 
adolescents dans l'espace de l'élève isolé. Le professeur va dans notre exemple, dans un premier temps se polariser sur l'action de «faire se regrouper » les jeunes, tout en injectant l'idée qu'ils sont dans un sentiment de consolation (puisque isolement est souvent synonyme de tristesse). Ensuite il va « jongler » entre la cohésion de groupe et le travail sur les contenus qu'il a choisi d'enseigner [4], la plupart du temps à l'aide de gestes tels que ceux de démonstration, de guidance, de maintien. Que l'on se trouve dans la recherche de cohésion mais surtout sur l'avancée des savoir-faire, le professeur place les adolescents dans un " milieu objectif » où les élèves agissent avec les connaissances développées en jeu [5]. On remarque clairement que les gestes font partie intégrante des ingéniosités du professeur, sans ces derniers, les différentes étapes des techniques ingénieuses ne pourraient progresser, l'enseignement voire l'apprentissage stagnerait. Nous pouvons ajouter que ces gestes reposent sur un système d'attentes puisque le professeur en use pour faire avancer les savoir-faire ou plus simplement pour que les adolescents agissent ensembles et ces derniers attendent du professeur ces gestes pour se sentir rassurés, tranquillisés.

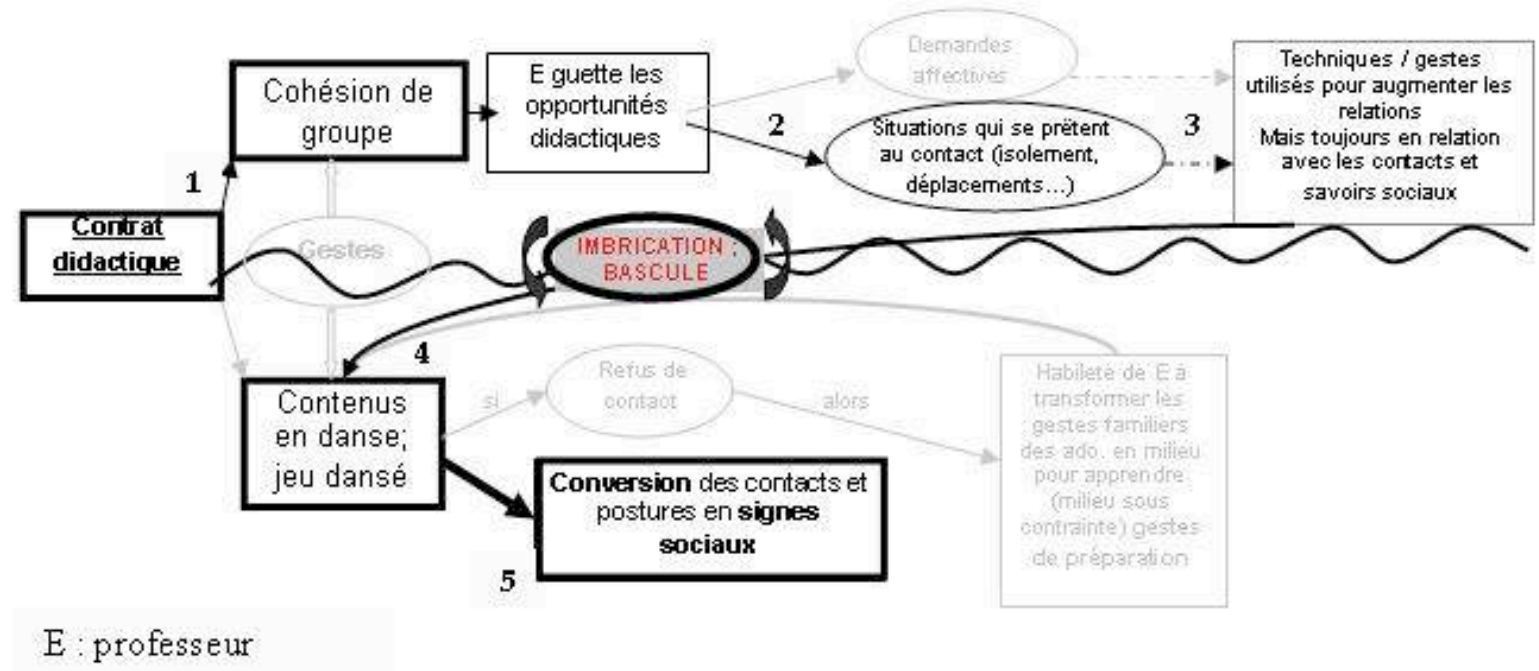

Figure 3: Présentation (pour ce qui est en gras) des actions et des focalisations du professeur liées à notre exemple

Nous nous sommes rendu compte que l'utilisation d'un comportement (d'isolement, de joie...), d'un ou plusieurs adolescents, souvent lié au handicap permet aux élèves de se sentir davantage concernés par les contenus. Est-ce le sens de l'action, des contacts qui est préservé ? ou la similitude avec les comportements quotidiens, qui fait que les jeunes avec autisme entrent et restent actifs plus facilement dans la situation ? Ceci paraît être primordial pour mettre les jeunes autistes en activité et d'autant plus pour escompter faire 
basculer une situation didactique en situation a-didactique (ce qui n'apparaît pas dans la figure 3). Si les interactions entre les adolescents fonctionnent, si le professeur sent que la situation a des potentialités a-didactiques, il va utiliser des gestes de type mise à distance pour optimiser la relation entre les adolescents concernés par la situation, voir les faire accéder à un moment dévolutif.

\section{Conclusion}

Cette recherche d'ingénierie didactique en danse avec un public atteint d'autisme, suggère que l'ingéniosité des gestes enseignants et le recours à des techniques adéquates par le professeur sont indispensables à l'avancée des capacités des adolescents voir même à la création de moments dévolutifs où apparaît chez eux un fonctionnement en responsabilité. La prédominance de la mise en place, ou de la redéfinition du contrat didactique, et de ce fait la dominance des situations didactiques, par rapport aux situations a-didactiques (présentes dans un milieu didactique, antagoniste et rétroactif), met en évidence l'effort constant du professeur à fixer des règles, des habitudes (de gestes, mouvements, postures) chez ces adolescents pour justement leur permettre par la suite d'être responsable d'une situation dite a-didactique. Ainsi avec ce public atteint du syndrome autistique, le contrat est continuellement à re-construire, à maintenir, à actualiser et ceci grâce en partie aux gestes du professeur, mais cela n'exclut pas la mise en place de milieux. En effet de réelles situations sont à réfléchir pour escompter stabiliser ou actualiser des capacités chez ces adolescents le professeur doit penser aux différents milieux (matériel, objectif) dans lesquels il place les jeunes autistes.

\section{Bibliographie}

Artigue, M. (1989). Une recherche d'ingénierie didactique sur l'enseignement des équations différentielles en premier cycle universitaire. Séminaire 107. IREM: Université Paris VII.

Artigue, M. (1990). L'ingénierie didactique, recherches en didactique des mathématiques. ( 9/3, pp.283-305). Grenoble : La Pensée Sauvage.

Baron-Cohen, S. (1995). La cécité mentale. Un essai sur l'autisme et la théorie de l'esprit. Grenoble : PUG.

Barthelemy, C., Hameury, L. \& Lelord, G. (1995). L'autisme de l'enfant : La Thérapie d'Echange et de Développement. Paris : Expansion Scientifique Française.

Brousseau G. (1998). Théorie des situations didactiques. Grenoble : La Pensée Sauvage. 
Brousseau G. (2002). Actant, Milieu. In Glossaire de quelques concepts de la théorie des situations didactiques en mathématiques. Définition 4.

Brousseau G. (2002). Contrat didactique. In Glossaire de quelques concepts de la théorie des situations didactiques en mathématiques. Définition 14.

Buten, H. (2003). Il y a quelqu'un là dedans : des autistes. Paris : Odile Jacob.

Frith U. (1992). L'énigme de l'autisme. Paris : Odile Jacob.

Jordan, R. \& Powell, S. (1997). Les enfants autistes. Les comprendre, les intégrer à l'école. Paris: Masson.

Loquet, M. (à paraître, 2007), Les techniques didactiques du professeur. In C. AmadeEscot (Eds.), Le didactique. Paris, France : Pour l'action, Editions Revue EP.S.

Loquet, M., Roncin, E., \& Roessle, S. (à paraître, 2006), L'action conjointe dans le système didactique en activités physiques, sportives et artistiques : les formes non verbales de communications didactiques. In G. Sensevy \& A. Mercier (Eds.), Agir Ensemble. L'action didactique conjointe du professeur et des élèves dans la classe. Rennes, France : PUR Presses Universitaires de Rennes.

Loquet, M., Roessle, S., \& Roncin, E. (2005). Les formes non verbales des communications didactiques chez l'intervenant en activités physiques, sportives et artistiques. In M.J. Perrin-Glorian \& Y. Reuter (Eds.), Les méthodes de recherches en didactiques. Acquisition et transmission des savoirs (pp. 45-58). Villeneuve d'Ascq, France : Presses Universitaires du Septentrion.

Perrin-Glorian M-J. \& Hersant M. (2003). Milieu et contrat didactique, outils pour l'analyse de séquences ordinaires. (23/2, pp.217-276). Recherche en didactique des mathématiques. Grenoble : La Pensée Sauvage.

Rabolini, C. , Rabolini, J.F., \& Suhner, J.J. (1995). L'espace thérapeutique des activités physiques et sportives. In F. Brunet, \& M. Caouette (Eds.), Corps et Psychiatrie Psychopédagogie des Activités Physiques et Sportives (pp. 138 ). Rennes: ENSP.

Sensevy, G. (à paraître, 2006). Des catégories pour décrire et comprendre l'action didactique. In G. Sensevy \& A. Mercier (Eds.), Agir Ensemble. L'action didactique conjointe du professeur et des élèves en classe. Rennes, France : PUR Presses Universitaires de Rennes. 